\title{
A Possible Way to Realize Controlled Nuclear Fusion at Low Temperatures
}

\author{
Shihao Chen' ${ }^{1}$, Ziwei Chen² \\ ${ }^{1}$ Northeast Normal University, Changchun, China \\ ${ }^{2}$ Beijing Jiaotong University, Beijing, China \\ Email: shchen@nenu.edu.cn,zwchen@bjtu.edu.cn
}

How to cite this paper: Chen, S.H. and Chen, Z.W. (2020) A Possible Way to Realize Controlled Nuclear Fusion at Low Temperatures. World Journal of Nuclear Science and Technology, 10, 23-31.

https://doi.org/10.4236/wjnst.2020.101003

Received: September 1, 2019

Accepted: November 22, 2019

Published: November 25, 2019

Copyright $\odot 2020$ by author(s) and Scientific Research Publishing Inc. This work is licensed under the Creative Commons Attribution International License (CC BY 4.0).

http://creativecommons.org/licenses/by/4.0/

(c) (i) Open Access

\begin{abstract}
This paper presents a new way to realize controlled nuclear fusion. The way is that a single energy neutron beam fuses with given nuclei, such as lithium nuclei or boron nuclei, so that the nuclear energy is released. The sort of fusion can be achieved at low temperatures, because a neutron has no charge and has a large reaction cross section with a nucleus. The fusion is easy to control and does not produce radioactive spent nuclear fuel. One of the five sorts of neutron sources is the electron neutron source in which a single energy electron beam collides with a single energy bare nucleus beam, such as the deuteron, to produce a single energy neutron. These neutrons irradiate target nuclei and are absorbed by the target nuclei, so that nuclear energy is released. Compared with conventional fusion, it has the disadvantage of releasing less energy and energy density. In addition, it takes a certain amount of energy to produce a beam of single-energy neutrons. However, if some of the input energy can be effectively recycled, the fusion process must produce more energy than the input energy.
\end{abstract}

\section{Keywords}

Nuclear Fusion at Low Temperature, Neutron Source, Lithium, Neutron-Rich Nucleus

\section{Introduction}

So far, controlled nuclear fusion has not realized. That's because not only does fusion need to be hotter than 100 million degrees, but it also needs to satisfy the Lawson conditions. For a 100 million degree plasma, the Lawson conditions are not easy to be satisfied. The fundamental reason is that there is a strong electrostatic repulsive potential between the positively charged bare nuclei. It is ob- 
vious that to reduce the nuclear fusion temperature is necessary.

Gamma ray has been produced in many laboratories. A scheme to generate gamma laser has also been proposed [1] [2] [3]. Based on this, [4] has proposed the idea which reduce the fusion temperature by using gamma rays or gamma lasers to irradiate the target nucleus so that the target nuclei are in an excited state. Different from this, this paper considers using neutrons to achieve nuclear fusion. Compared with conventional fusion, it has the disadvantage of releasing less energy and energy density. In addition, a certain amount of energy is required to produce a single-energy neutron beam. In order to output energy is larger than input energy, the thermal energy and light energy generated by the neutron beam generation process need to be effectively recycled. On the plus side, the fusion process can be done at low temperatures, easily controlled and no radioactive spent fuel to be produced.

\section{The Physical Mechanism of Controlled Fusion at Low Temperatures}

\subsection{The Fusion of Neutrons and Nuclei}

If the combining mode $\mathrm{A}$ of a nucleon system has higher energy than another combining mode $B$, when the nucleon system changes from A combining mode to $B$, there must be some nuclear energy to be released. Regardless of nuclear fission, conventionally designed nuclear fusion, or controlled fusion at low temperatures here, the physical principle of nuclear energy release is the same. The principle of controlled fusion at low temperatures is that a single energy neutron beam fuses with given nuclei so that the nuclear energy is released. The sort of fusion can be achieved at low temperatures, because a neutron has no charge and has a large reaction cross section with a nucleus, moreover, neutrons can be produced without protons colliding with nuclei or nuclei colliding with nuclei, so that the entire process of nuclear fusion does not have to overcome the electrostatic potential energy between the nuclei. A neutron can react with almost any nuclear. There are many kinds of nuclei which release nuclear energy after they absorb neutrons. For example,

$$
\begin{aligned}
& \mathrm{n}+{ }^{6} \mathrm{Li} \rightarrow \alpha+{ }^{3} \mathrm{~T}+4.78 \mathrm{MeV}, \quad \sigma_{n L i}=936 b, \\
& \mathrm{n}+{ }^{10} \mathrm{~B} \rightarrow \alpha+{ }^{7} \mathrm{Li}+2.792 \mathrm{MeV}, \quad \sigma_{n B}=3840 b,
\end{aligned}
$$

where $\sigma_{n L i}$ and $\sigma_{n B}$ are the inelastic scattering cross sections of a thermal neutron with ${ }^{6} \mathrm{Li}$ and ${ }^{10} \mathrm{~B}$, respectively.

\subsection{Neutron Sources}

There are the five sorts of neutron sources which are the spallation, the reactor, the spontaneous emission, the electron and the gamma ray neutron sources. The latter three sorts of neutron sources are considered for use in this controlled fusion at low temperatures. The physical mechanism of electron neutron sources is as follow. In a vacuum chamber, neutron-rich atoms are dissociated into electrons and bare nuclei. The electrons and bare nuclei are separated by electric 
field and magnetic field according to traditional technology, and are respectively modulated into a single-energy electron beam and a single-energy bare nucleus beam. The directions of the single-energy electron beam and the single-energy bare nucleus beam are opposite from each other. The kinetic energy of the electron relative to the bare nucleus is greater than the binding energy of the last neutron of the bare nucleus. As a result, after the electron collides with the bare nucleus, the neutron-rich bare nucleus will be broken into several daughter nuclei and neutrons due to electromagnetic and weak interactions. The electromagnetic interaction is clearly predominant here. The energies of the released neutrons are different from each other when the velocities of the single-energy electron beam relative to single-energy bare nucleus beam are different. Neutrons can be released out from many sorts of neutron-rich nuclei when electrons collide with the neutron-rich nuclei. For example, there is the following reaction when the kinetic energy of an electron relative to a deuteron is greater than the binding energy of a deuteron $2.224 \mathrm{MeV}$,

$$
\begin{gathered}
\mathrm{e}^{-}+\mathrm{d} \rightarrow \mathrm{e}^{-}+\mathrm{p}+\mathrm{n}-2.224 \mathrm{MeV}, \\
\mathrm{e}^{-}+\mathrm{d} \rightarrow v_{\mathrm{e}}+2 \mathrm{n}-2.224 \mathrm{MeV} .
\end{gathered}
$$

There is the following reaction when the kinetic energy of an electron relative to the beryllium nucleus ${ }^{9} \mathrm{Be}$ is greater than the binding energy of the last neutron in a ${ }^{9} \mathrm{Be}$ nucleus $1.665 \mathrm{MeV}$,

$$
\begin{gathered}
\mathrm{e}^{-}+{ }^{9} \mathrm{Be} \rightarrow \mathrm{e}^{-}+{ }^{8} \mathrm{Be}+\mathrm{n}-1.665 \mathrm{MeV}, \\
{ }^{8} \mathrm{Be} \rightarrow 2 \alpha+0.092218 \mathrm{MeV}, T\left({ }^{8} \mathrm{Be}\right) / 2=0.07 \mathrm{fs} .
\end{gathered}
$$

Analogously, a neutron-rich nucleus can also release neutrons when it absorbs a gamma photon whose energy is greater than the binding energy of the last neutron of the rich nucleus. For example, when the energy of a gamma photon is larger than $2.224 \mathrm{MeV}$ there is the following reaction

$$
\gamma+\mathrm{d} \rightarrow \mathrm{p}+\mathrm{n} \text {. }
$$

when the energy of a gamma photon is larger than $1.665 \mathrm{MeV}$ there is the following reaction

$$
\gamma+{ }^{9} \mathrm{Be} \rightarrow{ }^{8} \mathrm{Be}+\mathrm{n} .
$$

Some radioactive elements can release neutrons. The elements can used as the spontaneous emission neutron sources. One of the spontaneous emission neutron sources is ${ }^{252} \mathrm{Cf}$, its half-life is $T / 2=2.645 a$, its neutron yield is $2.31 \times 10^{12} \mathrm{~s}^{-1} \cdot \mathrm{g}^{-1}$, its energy spectrum distribution is the maxwell distribution, $N(E)=C \sqrt{E} \exp \left(-E / E_{r}\right)$, here $E_{r}=1.453 \pm 0.017 \mathrm{MeV}$, and $C$ is a normalization constant, in which some neutrons can fuse with the target nuclei ${ }^{6} \mathrm{Li}$ and ${ }^{9} \mathrm{Be}$. It is obvious that the energies of released neutrons by different radioactive elements are different from each other.

\subsection{The Ratio of Nuclear Energy Released to Energy Consumed}

To sum up, it can be seen that the process of the fusion of neutrons and nuclei is 
as follows. First, a large number of single-energy neutrons need to be produced. Secondly, the single-energy neutrons are fused with the target nucleus. Let the energy really consumed in the first step be $E_{N}$, the energy released in the second be $E_{F}$, only when $S=E_{F} / E_{N}>1$, the fusion is meaningful.

Let the binding energy of the electrons and the nucleus of a neutron-rich atom be $E_{A}$. In order to dissociate this atom into its bare nucleus and its electrons, it is necessary to input the energy $\tilde{E}_{A}=E_{A} / \eta_{A}>E_{A}$, here $\eta_{A}$ is the efficiency of $\tilde{E}_{A}$. In the final phase, these electrons will recombine with the positive ions to form atoms and to release optical energy $E_{A O}$. In general, $\Delta E_{A}=\tilde{E}_{A}-E_{A}$ will transform to thermal energy. A part $\eta_{A O} E_{A O}$ of $E_{A O}$ and a part $\eta_{a} \Delta E_{A}$ of $\Delta E_{A}$ can be recycled, here $\eta_{A O}<1$ and $\eta_{a}<1$ are the efficiencies of $E_{A O}$ of $\Delta E_{A}$, respectively. Consequently, the energy actually consumed in the process is $E_{A C}=\tilde{E}_{A}-\eta_{A O} E_{A O}-\eta_{a} \Delta E_{A}$. It is possible $E_{A C}<E_{A}$.

Let the consumed energy be $E_{S}$ in the process to modulate electrons into a single-energy electron beam and bare nuclei into a single-energy bare nuclear beam, and the optical energy radiated by the electrons and the bare nuclei be $E_{S O}$ when they accelerate or decelerate in the process. One part of $E_{S}$ is dedicated to the single electron beam and the single bare nuclear beam, and the remaining part of $E_{S}$ is $E_{S O}$. A part of $E_{S O}$ can be recycled, so the actual energy consumed in this process is $E_{S C}=E_{S}-\eta_{S O} E_{S O}$, here $\eta_{S O}$ is the recycled efficiency of $E_{S O}$.

To sum up, it can be seen that the process of the fusion of neutrons and nuclei is as follows. First, dissociate neutron-rich atoms into electrons and bare nuclei. For example, electrolysis of heavy water $\mathrm{D}_{2} \mathrm{O}$ yields $\mathrm{D}_{2}$. Dissociation of the heavy hydrogen gives the deuterons and electrons. Secondly, to modulate the electrons into a single-energy electron beam and neutron-rich nuclei into a single-energy bare beam. The kinetic energy of the electron in the beam relative to the bare nucleus is greater than the binding energy of the last neutron of the bare nucleus. Thirdly, in the colliding zone, the single-energy electron beam collides with the single-energy bare nuclear beam. As a result, single-energy neutrons are released, such as the process described in (3). Fourthly, the single-energy neutrons irradiates the given target nuclei and fuse with the target nuclei. The target nuclei disintegrate and releases nuclear energy, such as the process described in (1)-(2).

Let the energy of the initial state of a nuclear system be $E_{I}$, and the energy of the final state be $E_{F}, E_{I F}=E_{I}-E_{F}$ is the energy released by the nuclear system when it transforms from the initial state to the final state. But this energy cannot be released directly. It is necessary to go through an intermediate process. Let the energy required to transform from this initial state to this final state be $E_{M}$. In this case, the energy difference between the initial state and the final state is still the same, i.e. $E_{I F}=\left(E_{I}+E_{M}\right)-\left(E_{F}+E_{M}\right)$. One part $E_{M N}=\eta_{N} E_{M}$ of $E_{M}$ is used to release neutrons and transformed into kinetic energy of the single-energy neutrons, while the remaining part $E_{M T}=\eta_{T} E_{M}$ of $E_{M}$ is transformed into heat and light energy. One part $E_{M T R}=\eta_{T R} E_{M T}$ of $E_{M T}$ can be re- 
cycled, and the remaining part $E_{M T D}=\eta_{T D} E_{M T}=E_{M T}-E_{M T R}$ of $E_{M T}$ is dissipated. To sum up, it can be seen that if the output energy $E_{I F}$ of this fusion process is greater than the dissipated energy, i.e. $E_{I F}>E_{M T D}$, $E_{F D}=E_{I F}-E_{M T D}>0$ is the net output of energy in the fusion process. If $E_{I F} \leq E_{M T D}$, there is no net output of energy. $E_{F D}$ depends on the specific technology of recycling. There possibly is net output of energy in the fusion of neutrons and nuclei.

For example, let the initial state be composed of a deuteron $d$ and a lithium nucleus ${ }^{6} \mathrm{Li}$, the final state be composed of $\alpha, \mathrm{p}$ and ${ }^{3} \mathrm{~T}$, the energy difference between the initial state and the final state is

$$
\begin{aligned}
& {\left[\left(m_{d}+m_{L i}\right)-\left(m_{\alpha}+m_{T}+m_{p}\right)\right] c^{2}} \\
& =[(2.14102+6.015123)-(4.002603+3.016050+1.007825)] \times 931.49432 \\
& =2.558815 \mathrm{MeV}
\end{aligned}
$$

This initial state of course can not be directly transformed to this final state. In order to realize this transformation the following intermediate physical processes are necessary. Deuterium molecules $\mathrm{D}_{2}$ are dissociated into electrons and deuterons. The electrons and the deuterons are modulated into single energy electron beam and single energy deuteron beam, respectively. The kinetic energy of an electron in the electron beam relative to the deuteron beam is greater than 2.224 $\mathrm{MeV}$. The electron beam collides with the deuteron beam in the collision zone, so that single energy neutrons are released. The neutrons irradiate the target nuclei ${ }^{6} \mathrm{Li}$ and fuse the nuclei ${ }^{6} \mathrm{Li}$. Thus, the initial state $\left(\mathrm{d}+{ }^{6} \mathrm{Li}\right)$ transforms into the final state $\left(\alpha+{ }^{3} \mathrm{~T}+\mathrm{p}\right)$ by the immediate state $\left(\mathrm{p}+\mathrm{n}+{ }^{6} \mathrm{Li}\right)$. The energy released in the transformation from the immediate state to the final state is

$$
\begin{aligned}
& {\left[\left(m_{p}+m_{n}+m_{L i}\right)-\left(m_{\alpha}+m_{T}+m_{p}\right)\right] c^{2}} \\
& =[(1.007825+1.008665+6.015123)-(4.002603+3.016050+1.007825)] \\
& \quad \times 931.49432 \\
& \simeq 4.783=(2.224+2.559) \mathrm{MeV} .
\end{aligned}
$$

In order to obtain thermal neutrons with single energy from $\mathrm{D}_{2} \mathrm{O}$, the input energy must be larger than the binding energy of deuteron. On the other hand, not all thermal neutrons can fuse with lithium nuclei. Therefore, the inputting energy for releasing a thermal neutron must be significantly larger than the binding energy of the deuteron. But as long as the recycling technology is good, there must be $E_{I F}>E_{M T D}$.

There is no fusion of protons and nuclei or nuclei and nuclei in the whole process, hence this fusion of neutrons and nuclei can realized at low temperatures. In principle it can be done at room temperature.

The velocities of single-energy electron beam and single-energy deuteron beam in the laboratory coordinate system are $v_{e}$ and $v_{d}$, respectively, and their directions are opposite from each other, Thus the velocity of electrons relative to the deuterons is 


$$
u_{e d}=\frac{v_{e}+v_{d}}{1+v_{e} v_{d} / c^{2}} .
$$

when the electron's kinetic energy relative to the deuteron is greater than 2.224 $\mathrm{MeV}, u_{e d} \geq 0.983 c$. In order to reduce energy dissipation, $v_{d} \leq 30 \mathrm{~m} / \mathrm{s}$ is taken. So $u_{e d} \simeq v_{e}$ and $v_{d} / v_{e} \leq 10^{-7}$.

Let $n_{d}$ be the number density of the deuteron in the laboratory, and in the case the inelastic scattering cross section of electron and deuteron be $\sigma_{e d 0}=\sigma_{e d 0}(\mathrm{e}+\mathrm{d} \rightarrow \mathrm{p}+\mathrm{n})$, and the length of the collision zone of electron and deuteron be $L_{e d}$, then when

$$
\begin{gathered}
\sigma_{e d 0} n_{d} L_{e d}=1, \\
n_{e} v_{e}=n_{d} v_{d}, \quad n_{d}=1 / \sigma_{e d 0} L_{e d}=10^{7} n_{e},
\end{gathered}
$$

the inelastic scattering probability of each incident electron with the deuteron is close to 1 . When $L_{e d}$ is very large, $\tilde{L}_{e d}=L_{e d} / k$ can been taken as the length of the collision zone, here $k$ is an integer. In this case, some electrons have no inelastic scattering with the deuteron. The electrons will be again transported into a speed regulating device, and then are transported back to the collision zone. In this process, these electrons are in accelerated motion and radiate light. This light, especially synchrotron radiation, can be recycled.

Let the cross section of single-energy deuteron beam be $S$, which is also the cross section of single-energy electron beam, then the deuteron current intensity and electron beam current intensity are respectively

$$
J_{d}=e^{+} n_{d} v_{d} S=-e^{-} n_{e} v_{e} S=-J_{e}
$$

A deuteron can release a neutron when it absorbs a photon with its energy to be larger than the bind energy of a deuteron $2.224 \mathrm{MeV}$. Thus, single energy neutrons can be obtained by gamma laser or single energy gamma photon beam to irradiate the deuterons. Let the inelastic scattering section of a such gamma photon with a deuteron be $\sigma_{\gamma d}$, then when

$$
\begin{gathered}
\sigma_{\gamma d} n_{d} L_{\gamma d}=1, \\
n_{d} v_{d}=n_{\gamma} c, v_{d} \leq 30 \mathrm{~m} / \mathrm{s}, n_{d} \geq 10^{7} n_{\gamma},
\end{gathered}
$$

where $L_{\gamma d}$ is the length of the collision zone of the gamma photons with the deuterons, and $n_{\gamma}$ is the number density of the gamma photons, the probability of each gamma photon being absorbed by the deuterons is close to 1 .

Using these single-energy neutrons to irradiate lithium or boron atoms, fusion reactions (1) or (2) will occur. Let the number density of ${ }^{6} \mathrm{Li}$ nuclei and ${ }^{10} \mathrm{~B}$ nuclei be $n_{L i}$ and $n_{B}$. lithium or boron atoms are placed in the annular cylinder around this thermal neutron source. The radius differences between the inner and outer cylinders are $L_{L i}$ and $L_{B}$, respectively, when

$$
\sigma_{n L i} n_{L i} L_{L i}=1, \sigma_{n B} n_{B} L_{B}=1
$$

The probability of each neutron to be absorbed of is close to 1 .

The binding energy of a deuterium is less $14 \mathrm{eV}$. Therefore, in the process of 
making a single energy electron beam and a single energy deuteron beam, the average actual energy dissipation of one deuteron and one electron is not more than $1 \mathrm{KeV}$. It can be seen from the above that if the probability of absorption of a single energy electron by deuterons is greater than 0.7 , and the probability of absorption of a single energy neutron by lithium atoms is greater than 0.9 , then the average net energy gained from the fusion process of one deuterium and one lithium is greater than $1 \mathrm{MeV}$.

\section{A Device for Fusion of Neutrons with Nuclei}

This fusion device consists mainly of the following parts. A vacuum colliding zone of a single energy electron beam with a single energy nucleus beam composed of neutron-rich bare nuclei, the input and output channels of electrons, the input and output channels of neutron rich nuclei, a device dissociating neutron-rich atoms into the electrons and the neutron-rich nuclei, a device of modulating electronic velocities, a device modulating velocities of neutron-rich nuclei, a target atom container $T$, the channels of the target atoms to be imported into $T$ and to be out exported from $T$, a device separating ions, electrons and target atoms, and energy transfer device.

The colliding zone is a cylinder $C$, which is parallel to the $X$-axis, with a length of $L$ and a diameter of $r$, two open ends, and surrounded by thin walls which can withstand high temperature and allow neutrons to pass through smoothly. Electric and magnetic fields parallel to the $X$-axis are distributed in the colliding zone. In the colliding region $C$, the single energy electron beam collides with the single energy neutron-rich nuclear beam, so that single energy neutrons are released. Here the kinetic energy of an electron relative to the neutron-rich nucleus is greater than the final binding energy of the neutron in the rich nucleus.

The target atoms container $T$ is composed of an outer cylinder, an inner cylinder and two sides. The outer cylinder is a cylinder with radius $R$, length $L$ and coaxial line with the colliding zone $C$, made of lead plate absorbing neutrons. The inner cylinder is just cylinder $\mathrm{C}$. The container $T$ is filled with target atoms whose nuclei will fuse with neutrons. The two sides of the container $T$ connect the pipe importing the target atoms into $T$ and the pipe exporting the target atoms from $T$, respectively.

The target atoms flow into the container $T$ at the required velocity $v$ through the inlet of the container $T$, and flows out of the container $T$ at the same velocity $v$ through the outlet. The target atoms which have absorbed the neutrons break into several daughter nuclei and electrons. These particles flow out of the container $T$ and then flow into the separation device of the ions, electrons and target atoms in which there is a given magnetic field. Under the action of the magnetic field, these daughter nuclei and electrons are respectively concentrated to form the positive and negative poles of the power. The target atoms which doesn't absorb the neutrons are again transported to the container $T$.

At both ends of the colliding zone $C$ along the $Y$ direction there are electron importing and exporting pipelines and the importing pipelines of neutron-rich 
nuclei, respectively. There is magnetic field in the $z$ direction of the pipelines. Under the magnetic field, the daughter nuclei are separated and then recombined with the electrons to form atoms and to release energy. The atoms are collected after cooling.

Under action of the magnetic field, the neutron-rich nuclei without absorbing neutrons are separated and then input into the velocity modulation device of neutron-rich nuclei. Under action of the magnetic field, the electrons are separated and then input into the electron velocity modulating device.

There are the given electronic field and magnetic field in the electronic velocity modulating device. The electrons are decomposed into multiple beams according to the velocities of the electrons. Then electrons with different velocities are modulated into the same single-energy electron beam. The same is true for the velocity modulating device of neutron-rich nuclei. The device dissociating neutron-rich atoms dissociates neutron-rich atoms into bare nuclei and electrons. The electrons and the neutron-rich bare nuclei are transported to the electron velocity modulating device and the velocity modulating device of neutron-rich nuclei, respectively.

The single energy electron beam and the single energy beam of neutron-rich nuclei are imported into the colliding zone, respectively.

The energy transmission system absorbs and transmits the energy released by fusion and the heat and the light energy converted from the input energy.

\section{Conclusion}

The mechanism of this sort of controlled nuclear fusion is that single energy neutrons fuse with given nuclei, such as ${ }^{6} \mathrm{Li}$ or ${ }^{10} \mathrm{~B}$, to release nuclear energy. One of the five types of neutron sources is the electron neutron source, where a single energy electron beam collides with a single energy bare nuclear beam (such as deuterium) to produce a single energy neutrons. These neutrons irradiate the target nucleus and are absorbed by the target nucleus so that nuclear energy is released. Compared with conventional nuclear fusion, it has the disadvantages of low energy release and low energy density. In addition, it takes a certain amount of energy to produce a beam of single-energy neutrons. However, if some of the input energy can be effectively recycled, the fusion process must produce more energy than the input energy. On the plus side, this fusion process can be done at low temperatures (in principle, at room temperature), because a neutron has no charge and has a very large cross section with nuclei. The fusion is easy to control and does not produce radioactive spent nuclear fuel.

\section{Acknowledgements}

This work is supported by National Natural Science foundation of China 11075064. We are very grateful for the fund's support.

\section{Conflicts of Interest}

The authors declare no conflicts of interest regarding the publication of this paper. 


\section{References}

[1] Chen, S.H. and Chen, Z. (2014) Electron-Photon Backscattering Laser. Laser Physics, 24, 045805. https://doi.org/10.1088/1054-660X/24/4/045805

[2] Chen, Z. and Chen, S.H. (2015) A Discussion on Electron-Photon Backscattering Lasers and Electron-Photon Backscattering Laser in a Laser Standing Wave Cavity. Laser Physics, 4, 045803. https://doi.org/10.1088/1054-660X/25/4/045803

[3] Chen, S.H. and Chen, Z. (2016) Coherent Conditions of Electron-Photon Backscattering Light in a Wiggle Magnetic Field. Laser Physics, 26, 025807. https://doi.org/10.1088/1054-660X/26/2/025807

[4] Chen, S.H. and Chen, Z. (2018) A Way to Realize Controlled Nuclear Fusion by $\gamma$-Laser or $\gamma$-Ray. World Journal of Nuclear Science and Technology, 8, 190-196. https://doi.org/10.4236/wjnst.2018.84016 